\title{
Elastase-mediated phosphatidylserine receptor cleavage impairs apoptotic cell clearance in cystic fibrosis and bronchiectasis
}

\author{
R. William Vandivier, ${ }^{1}$ Valerie A. Fadok, ${ }^{2}$ Peter R. Hoffmann, ${ }^{2}$ Donna L. Bratton, ${ }^{3}$ \\ Churee Penvari, ${ }^{4}$ Kevin K. Brown, ${ }^{5}$ Joseph D. Brain, ${ }^{6}$ Frank J. Accurso, ${ }^{4}$ \\ and Peter M. Henson ${ }^{2}$
}

${ }^{1}$ Division of Pulmonary Sciences and Critical Care Medicine, Department of Medicine, University of Colorado
Health Sciences Center, Denver, Colorado, USA
${ }^{2}$ Program in Cell Biology, Department of Pediatrics, and
${ }^{3}$ Department of Pediatrics, National Jewish Medical and Research Center, Denver, Colorado, USA
${ }^{4}$ Department of Pediatrics, University of Colorado Health Sciences Center, Mike McMorris Cystic Fibrosis Center,
Children's Hospital, Denver, Colorado, USA
${ }^{5}$ Department of Medicine, National Jewish Medical and Research Center, Denver, Colorado, USA
${ }^{6}$ Physiology Program, Department of Environmental Health, Harvard School of Public Health, Boston, Massachusetts, USA

Address correspondence to: R. William Vandivier, National Jewish Medical and Research Center, 1400 Jackson Street, Room D505, Denver, Colorado 80262, USA.

Phone: (303) 398-1380; Fax: (303) 398-1381; E-mail: vandivierb@njc.org.

Received for publication June 20, 2001, and accepted in revised form December 17, 2001.

Cystic fibrosis is characterized by an early and sustained influx of inflammatory cells into the airways and by release of proteases. Resolution of inflammation is normally associated with the orderly removal of dying apoptotic inflammatory cells through cell recognition receptors, such as the phosphatidylserine receptor, CD36, and $\alpha v$ integrins. Accordingly, removal of apoptotic inflammatory cells may be impaired in persistent inflammatory responses such as that seen in cystic fibrosis airways. Examination of sputa from cystic fibrosis and non-cystic fibrosis bronchiectasis patients demonstrated an abundance of apoptotic cells, in excess of that seen in patients with chronic bronchitis. In vitro, cystic fibrosis and bronchiectasis airway fluid directly inhibited apoptotic cell removal by alveolar macrophages in a neutrophil elastase-dependent manner, suggesting that elastase may impair apoptotic cell clearance in vivo. Flow cytometry demonstrated that neutrophil elastase cleaved the phosphatidylserine receptor, but not CD36 or CD32 (Fc $\gamma \mathrm{RII})$. Cleavage of the phosphatidylserine receptor by neutrophil elastase specifically disrupted phagocytosis of apoptotic cells, implying a potential mechanism for delayed apoptotic cell clearance in vivo. Therefore, defective airway clearance of apoptotic cells in cystic fibrosis and bronchiectasis may be due to elastase-mediated cleavage of phosphatidylserine receptor on phagocytes and may contribute to ongoing airway inflammation.

J. Clin. Invest. 109:661-670 (2002). DOI:10.1172/JCI200213572.

\section{Introduction}

Resolution of acute inflammation is normally accomplished by removal of dying, apoptotic inflammatory cells $(1,2)$. In the cystic fibrosis (CF) lung, inflammation is persistent and necrotic cells accumulate in the airway (3), suggesting that normal apoptotic cell removal mechanisms may be impaired. Recognition of apoptotic cells promotes the resolution of inflammation in a number of ways. Phagocytosis of apoptotic cells leads to their efficient removal before disruption of the plasma membranes and spillage of potentially harmful intracellular contents. Removal of apoptotic cells also actively suppresses ongoing inflammation by the production of antiinflammatory signals such as TGF- $\beta$ (4). These antiinflammatory signals inhibit inflammatory mediator production and subsequently enhance resolution of the response $(4,5)$.
Apoptotic cells target themselves for recognition and uptake into phagocytes by expressing surface ligands, in particular phosphatidylserine (PS) (6, 7). A variety of receptors are involved in the removal process, especially a recently cloned specific receptor for PS (8). Other recognition molecules include CD36 (9), other scavenger receptors (10), CD14 (11), a variety of integrins $(12,13)$, as well as soluble bridging molecules such as thrombospondin (9), the collectins $(14,15)$, or $\mathrm{C} 1 \mathrm{q}(15$, 16). However, the requirement for PS expression $(6,7)$ and a dominant role for the PS receptor (8) suggest that this interaction plays a major role in the recognition and uptake of apoptotic cells. The PS receptor also appears critical in the anti-inflammatory effects of apoptotic cell recognition and initiation of the TGF- $\beta$ production, both in vitro $(4,5)$ and in vivo $(17)$.

The clearance of dying apoptotic cells from developing tissues or sites of inflammation is normally a 
highly efficient process. Examples include the removal of apoptotic cells from the limb during embryonic development, shrinkage of the tadpole tail during metamorphosis, and loss of apoptotic thymocytes during normal or steroid-induced involution of the thymus (1). Despite significant ongoing apoptosis, remarkably few apoptotic cells can be detected in these tissues at any point in time due to their rapid clearance (1). In the human respiratory tract, cell removal during resolution of inflammation is just as efficient. During the acute respiratory distress syndrome neutrophils (PMNs) migrate into the lung in large numbers reaching $7.4 \times 10^{5} / \mathrm{ml}(18)$; however, only $1.6 \%$ of these are apoptotic (18). Similarly, in communityacquired pneumonia PMNs reach $1.1 \times 10^{6} / \mathrm{ml}$ (19), yet only $0.3 \%$ are apoptotic (20). This suggests that extrusion of inflammatory cells into the airway lumen, by itself, is not a sufficient cause for the accumulation of apoptotic cells.

Accordingly, detection of significant numbers of apoptotic cells in an inflammatory site, such as the airways of patients with CF and non-CF bronchiectasis, may suggest dysregulated clearance. Abnormal removal of apoptotic inflammatory cells in CF could, in turn, facilitate the persistence or enhancement of airway inflammation by (a) allowing the development of postapoptotic necrosis with the consequent release of proinflammatory materials and by (b) altering mechanisms that would normally actively suppress proinflammatory mediator production.

In CF airways, inflammation begins during the neonatal period, extends through adulthood, and is associated with chronic bacterial infection, increased IL-8, TNF- $\alpha$, PMN influx, and release of PMN proteases (21-23). Evidence of airway inflammation has been demonstrated in infants as young as 4 weeks, and a significant proportion of infants under 1 year of age have airway inflammation in the absence of infection (21). Regardless of the initiating stimulus, the result is massive influx of inflammatory cells into the airways, where they die, liberate intracellular proteases that overwhelm antiprotease defenses (23), and leave proteases unopposed to injure airways and impair host defense. This complex inflammatory milieu in the CF airway may be considered an extreme manifestation of a pattern of protease/antiprotease imbalance that appears to be representative of a variety of chronic airway inflammatory diseases, such as bronchiectasis (24), chronic bronchitis $(25,26)$, asthma (26), and $\alpha 1$-antitrypsin deficiency (27).

We sought to determine whether apoptotic cells accumulate excessively in the airways of CF patients compared with other diseases characterized by chronic airway inflammation and find that airway apoptotic cells are markedly increased in patients with CF and non-CF bronchiectasis. We further show an impairment of apoptotic cell engulfment induced by fluid from such airways and demonstrate that this may be due to the cleavage of the PS receptor from phagocytic cells by active elastase.

\section{Methods}

Materials. Purified human elastase (21 U/mg protein), human cathepsin $\mathrm{G}(3 \mathrm{U} / \mathrm{mg}$ protein), and the specific elastase inhibitor, MeOSuc-Ala-Ala-Pro-Val-CMK(NEI), were obtained from Calbiochem-Novabiochem Corp. (San Diego, California, USA). The specific cathepsin G inhibitor (CGI), Z-Gly-Leu-Phe-CMK, was obtained from Enzyme Systems Products Inc. (Livermore, California, USA). DMP777, a specific inhibitor of PMN elastase, and its inactive enantiomer, SJ527, were kindly provided by DuPont Pharmaceuticals Co. (Wilmington, Delaware, USA). Human proteinase 3 (13.5 U/mg protein) was obtained from Elastin Products (Owensville, Missouri, USA). Mouse monoclonal anti-PS receptor IgM was prepared in this laboratory as described previously (8). Ab's obtained from PharMingen (San Diego, California, USA) included mouse monoclonal antihuman CD36 IgM, mouse monoclonal anti-human CD45 IgG, mouse monoclonal anti-human CD32 (FcyRII) IgG, mouse monoclonal anti-keyhole limpet hemocyanin $\operatorname{IgM}$, and mouse IgG. Ab's obtained from Jackson ImmunoResearch Laboratories Inc. (West Grove, Pennsylvania, USA) included rabbit anti-mouse IgG, Cy-3 goat IgG anti-mouse IgM, and Cy-3 goat IgG anti-mouse IgG. Human $\alpha 1$-antitrypsin was obtained from Sigma Chemical Co. (St. Louis, Missouri, USA).

Human subjects. The study was approved by, and performed in accordance with, the ethical standards of our institutional review boards on human experimentation. Written informed consent was obtained from each subject. Chronic bronchitis patients were current smokers or had smoked previously and had sputum production for at least 3 months during 2 successive years. Bronchiectasis was documented in patients using high-resolution computed tomography, and none were heterozygous, or homozygous, for 86 known CF mutations (Genzyme Corp., Framingham, Massachusetts, USA). Bronchiectasis patients included four with a history of Mycobacterium avium intracellulare, one with allergic bronchopulmonary aspergillosis, and one with idiopathic bronchiectasis. All CF patients were homozygous for the $\Delta F 508$ CF transmembrane regulator mutation, except one who was a compound heterozygote for $\Delta \mathrm{F} 508 / \mathrm{R} 553 \mathrm{X}$.

Sputum was collected from patients in all three groups during a clinical exacerbation defined by increased cough and sputum production. Sputum cultures were taken to identify bacterial or fungal pathogens. All patients had spirometry within a month of the study.

Assessment of sputum apoptotic cells. First morning sputum was collected on ice and processed immediately with Sputolysin (Caldon Biotech Inc., Carlsbad, California, USA), centrifuged onto slides (Cytospin; Shandon Inc., Pittsburgh, Pennsylvania, USA), stained with modified Wright's Giemsa (Fisher Scientific, Pittsburgh, Pennsylvania, USA), and analyzed blindly for apoptosis using nuclear condensation or a modified TUNEL assay per the manufacturer's recommendations (Promega Corp., Madison, Wisconsin, USA). Sputum alveolar macrophages (AMs) were also examined 
blindly for ingestion of apoptotic cells and quantitated using a phagocytic index, as described previously (6). Some samples were assessed for apoptosis using transmission electron microscopy (Philips 400T twin lens; Philips, Eindhoven, The Netherlands).

Preparation of CF and non-CF bronchiectasis sol. Sol was made from $\mathrm{CF}$ or non-CF bronchiectasis sputum using a protocol described previously (28). First morning sputa were collected on ice from hospitalized patients and fractionated immediately into aqueous sol and gel fractions by ultracentrifugation $50,000 \mathrm{~g}$ for $90 \mathrm{~min}$ utes). CF sol was pooled from six patients, and non-CF bronchiectasis sol was pooled from three patients. Elastase and cathepsin $\mathrm{G}$ activity were measured using methods that depended on elastase cleavage of $\mathrm{MeO}$ Suc-Ala-Ala-Pro-Val-p-nitroanilide (Calbiochem-Novabiochem Corp.) and cathepsin G cleavage of Suc-AlaAla-Pro-Phe- $p$-nitroanilide (Calbiochem-Novabiochem Corp.), as described previously (28).

Cell isolation and culture. Human monocyte-derived macrophages (HMDMs) and PMNs were isolated using Percoll gradient centrifugation, as described previously (2). Monocytes were plated in 24-well tissue culture plates (Becton Dickinson and Co., Franklin Lakes, New Jersey, USA), and matured to macrophages by culturing in X-vivo medium (BioWhittaker Inc., Walkersville, Maryland, USA) containing $10 \%$ human serum pooled from five donors, at $37^{\circ} \mathrm{C}$ in $10 \% \mathrm{CO}_{2}$ for 6-8 days. Approximately $5 \times 10^{5} \mathrm{HMDMs}$ were present in each well.

Human AMs were isolated from normal subjects. Bronchoscopy was performed and subjects were lavaged with $240 \mathrm{ml} \mathrm{0.9 \%} \mathrm{saline} \mathrm{at} \mathrm{room} \mathrm{temperature.}$ AMs were resuspended in X-vivo (BioWhittaker Inc.) medium, cultured in a 96-well plate at $10^{5}$ cells/well at $37^{\circ} \mathrm{C}$ in $5 \% \mathrm{CO}_{2}$ for 30 minutes, washed to remove nonadherent cells, and then cultured for 2 hours prior to experimentation.

Human T lymphocyte Jurkat cells were obtained from the American Type Culture Collection (Manassas, Virginia, USA), and human myelomonocyte PLB985 cells were a kind gift from Christina Leslie (National Jewish Medical and Research Center, Denver, Colorado, USA). Both cell lines were cultured in RPMI (MediaTech Inc., Herndon, Virginia, USA) with 10\% heat-inactivated FBS (Gemini Bio Products, Calabasas, California, USA) and supplemented with $2 \mathrm{mM}$ L-glutamine, 100 $\mathrm{U} / \mathrm{ml}$ penicillin, and $100 \mu \mathrm{g} / \mathrm{ml}$ streptomycin (Sigma Chemical Co.).

Induction of apoptosis. Jurkat cells or PLB985 cells were exposed to ultraviolet irradiation at $254 \mathrm{~nm}$ for 10 minutes or 5 minutes, respectively, and cultured for 3.5 hours (Jurkat cells) or 2 hours (PLB985 cells). Human PMNs were exposed to ultraviolet irradiation at $254 \mathrm{~nm}$ for 10 minutes and cultured for 2.5 hours in RPMI with 1\% low-endotoxin BSA (Sigma Chemical Co.). All cells were approximately $70 \%$

\section{Table 1}

apoptotic by nuclear condensation at the time of experimentation, as described previously (2).

Phagocytosis assays. Six- to eight-day-old HMDMs were cocultured with apoptotic Jurkat cells or PLB985 cells suspended in $500 \mu \mathrm{l} \mathrm{X}$-vivo (BioWhittaker Inc.) at $37^{\circ} \mathrm{C}$ in $10 \% \mathrm{CO}_{2}$ in the absence of human serum for 1 hour at a ratio of 5:1 (apoptotic cell to HMDM). In some experiments viable or IgG-opsonized cells (Jurkat cells only) were used as the target cell. Jurkat cells were opsonized with mouse monoclonal anti-human CD45 IgG followed by rabbit polyclonal anti-mouse $\operatorname{IgG}$ as described previously (6). HMDMs were then gently washed with HBSS to remove uningested cells, fixed, and stained with a modified Wright's Giemsa stain (Fisher Scientific). Phagocytosis was determined by visual inspection of samples and was expressed as a phagocytic index (6). Each condition was tested in duplicate and a minimum of 400 HMDMs was counted blindly. HMDMs were greater than $99 \%$ viable in the presence and absence of $\mathrm{CF}$ sol, assessed by trypan blue exclusion. Fresh AMs were cocultured with apoptotic PMNs in a similar fashion, except experiments were conducted in 96-well plates.

Reconstitution of plasma membrane outer leaflet with PS. PLB985 cell membranes were reconstituted with lipids using liposomes containing a 50:50 molar ratio of PS to phosphatidylcholine (PC) (both from Avanti Polar Lipids Inc., Alabaster, Alabama, USA), or 100\% PC, as previously described, and used immediately (7).

FACS analysis. HMDMs or AMs were suspended in HBSS containing 2\% FBS (Gemini Bio Products). HMDMs or AMs $\left(10^{6}\right)$ were incubated with $1 \mu \mathrm{g}$ of primary $\mathrm{Ab}$ on ice for 30 minutes, washed twice, then incubated with secondary $\mathrm{Ab}$ (1:50 dilution) on ice for 30 minutes. Washed macrophages were analyzed on a FACScan cytometer using PCLysys software (Becton Dickinson and Co.).

Statistics. The means were analyzed using ANOVA for multiple comparisons; when ANOVA indicated significance, the Tukey-Kramer honestly significant difference (HSD) test for all pairs was used to compare groups (Table 1, Figure 1, e and f, and Figure 2, a and c). For all

Patient demographics

\begin{tabular}{|c|c|c|c|}
\hline & Chronic bronchitis & Bronchiectasis & $\mathrm{CF}$ \\
\hline & 6 & 6 & 6 \\
\hline Age (year) & $65.5 \pm 2.7$ & $63.5 \pm 4.7$ & $18.3 \pm 1.6^{\mathrm{A}}$ \\
\hline $\mathrm{FEV}_{1}(\mathrm{I})$ & $0.9 \pm 0.1$ & $1.3 \pm 0.1$ & $1.8 \pm 0.2^{\mathrm{B}}$ \\
\hline $\mathrm{FVC}(\mathrm{I})$ & $2.2 \pm 0.4$ & $2.0 \pm 0.1$ & $2.8 \pm 0.2$ \\
\hline $\mathrm{FEV}_{1} / \mathrm{FVC}(\%)$ & $43.8 \pm 6.8$ & $63.3 \pm 5.0$ & $63.5 \pm 6.5$ \\
\hline Pseudomonas aeruginosa (mucoid) & 0 & 1 & $6^{A}$ \\
\hline Pseudomonas aeruginosa (nonmucoid) & 0 & 2 & 1 \\
\hline Staphylococcus aureus & 1 & 0 & 2 \\
\hline Micobacterium avium intracellulare & 0 & $4^{C}$ & 0 \\
\hline
\end{tabular}

ASignificantly different from chronic bronchitis and bronchiectasis groups $(P<0.05)$. ${ }^{B} \mathrm{Sig}-$ nificantly different from chronic bronchitis group $(P<0.05)$. ${ }^{C}$ Significantly different from chronic bronchitis and CF groups $(P<0.05)$. FEV ${ }_{1}$, forced expiratory volume in 1 second; FVC, forced vital capacity. 

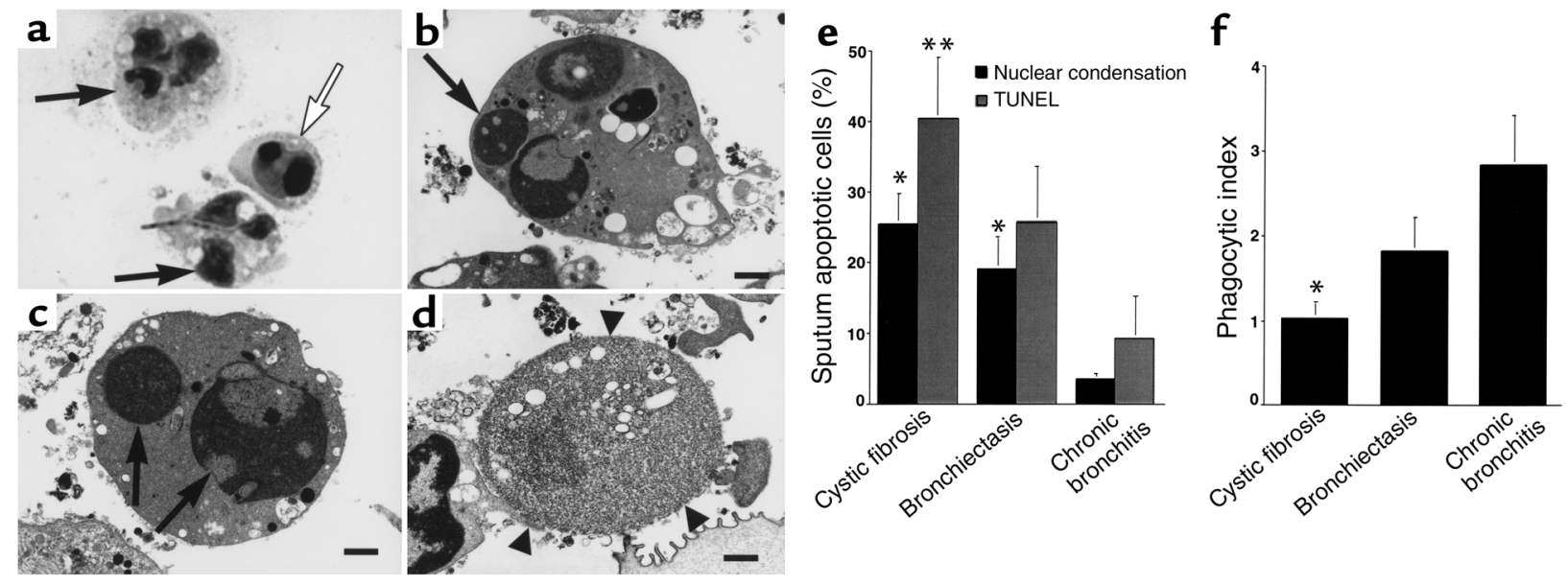

Figure 1

Clearance of apoptotic cells is defective in CF airways. (a) Wright's Giemsa stain of CF sputum ( $\times 100)$ showing apoptotic cells with condensed nuclei (open arrow) and normal PMNs (arrows). (b-d) Transmission electron micrograph of sputum from a CF patient ( $\times 6,300$; bars: $1 \mu \mathrm{m}$ ) demonstrating early apoptosis $(\mathbf{b})$, late apoptosis $(\mathbf{c})$, and postapoptotic necrosis (d). Arrows $(\mathbf{b}$ and $\mathbf{c})$ indicate condensed apoptotic nuclei. Arrowheads (d) show loss of membrane integrity during necrosis. (e) Sputa from CF or bronchiectasis patients contain more apoptotic cells compared with chronic bronchitis. The percentage of apoptotic cells (by nuclear condensation and TUNEL staining) \pm SEM is shown for six patients per group. *Apoptosis by nuclear condensation is significantly different from chronic bronchitis $(P<0.05)$. * *Apoptosis by TUNEL staining is significantly different from chronic bronchitis $(P<0.05)$. (f) Airway macrophages from CF patients ingest fewer apoptotic cells. The mean phagocytic index of sputum macrophages \pm SEM is shown for six patients per group. *Phagocytic index is significantly different from chronic bronchitis $(P<0.05)$.

other experiments in which an internal control was present, the Dunnett method was used. All data were analyzed using JMP (version 3) Statistical Software for the Macintosh (SAS Institute Inc., Cary, North Carolina, USA) and are presented plus or minus SEM.

\section{Results}

Study patients. Patient demographics are summarized in Table 1. CF patients were younger, had less expiratory airflow obstruction, as measured by the forced expiratory volume in 1 second $\left(\mathrm{FEV}_{1}\right)$, and were more likely to be colonized with mucoid Psendomonas aeruginosa. Bronchiectasis patients were more likely to have been infected with M. avium intracellulare.

Sputum apoptotic cells are increased in patients with CF and non-CF bronchiectasis. Apoptotic cells were quantitated in sputum by nuclear condensation (Figure 1a) and by TUNEL staining. Transmission electron microscopy demonstrated the presence of early apoptotic, late apoptotic, and necrotic PMNs in CF sputum (Figure 1, b-d). Sputum apoptotic cells were present in all patient groups (Figure 1e). Assessed by nuclear condensation, apoptotic cells were greater in $\mathrm{CF}$ and non-CF bronchiectasis compared with chronic bronchitis patients. TUNEL staining confirmed these results; however, the difference between bronchiectasis and chronic bronchitis did not reach significance. Sputum samples were also examined for AM ingestion of apoptotic cells (Figure 1f). CF AMs contained fewer apoptotic bodies than those from patients with chronic bronchitis. These data establish that apoptotic cells are present in the airways of patients with CF, non-CF bronchiectasis, and chronic bronchitis during clinical exacerbations, but the relative abundance of apoptotic cells depends upon the underlying disease. Furthermore, clearance of apoptotic cells by CF AMs appears to be defective.

CF sol impairs macrophage phagocytosis of apoptotic cells in an elastase-dependent manner. Experiments were conducted to test whether CF airway fluid impaired the ability of HMDMs to ingest apoptotic cells (Figure 2a). These experiments demonstrated that ingestion of apoptotic Jurkat cells by HMDMs, but not IgG-opsonized or viable Jurkat cells, was inhibited by $10 \%$ CF sol.

Serine proteases, present in the airway secretions of patients with CF and non-CF bronchiectasis, were considered as possible contributors to the sol-mediated delay in apoptotic cell clearance. CF sol contained $3 \mathrm{U} / \mathrm{ml}$ of PMN elastase and $28 \mathrm{mU} / \mathrm{ml}$ of cathepsin G. Non-CF bronchiectasis sol contained 10 $\mathrm{U} / \mathrm{ml}$ of PMN elastase and $77 \mathrm{mU} / \mathrm{ml}$ of cathepsin $\mathrm{G}$. Two specific inhibitors of PMN elastase, DMP777 (20 $\mu \mathrm{M})$ and NEI $(100 \mu \mathrm{M})$, completely and specifically inhibited elastase activity in 10\% CF and bronchiectasis sol (data not shown). In addition, a specific inhibitor of cathepsin G, CGI $(10 \mu \mathrm{M})$, completely and specifically inhibited cathepsin $\mathrm{G}$ activity in both sol preparations (data not shown).

The specific PMN elastase inhibitor, DMP777, was used to test whether elastase contributed to the inhibitory effect of CF sol (Figure 2b). HMDMs cultured with apoptotic Jurkat cells, in the presence of CF sol, ingested fewer apoptotic cells, but not in the presence of DMP777. Neither the inactive enantiomer, SJ527, nor the methylcellulose vehicle prevented the CF sol-mediated decrease in apoptotic cell ingestion. Similarly, pretreatment of HMDMs with $10 \%$ CF sol 

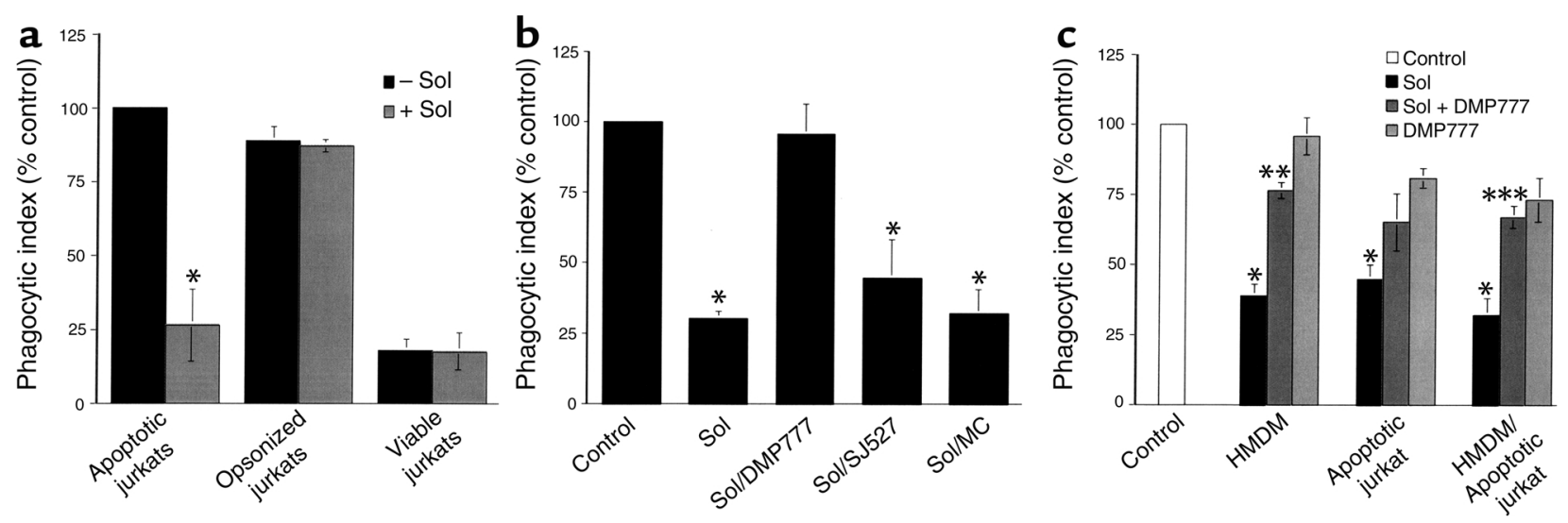

Figure 2

CF sol inhibits HMDM ingestion of apoptotic but not IgG-opsonized Jurkat cells in an elastase-dependent manner. (a) HMDMs were cocultured with apoptotic, opsonized, or viable Jurkat cells in the presence or absence of $10 \%$ CF sol. The mean phagocytic index as percentage of control \pm SEM is shown for three replicates per group. Control mean phagocytic index: $28.6 \pm 4.4$. *Significantly different from apoptotic Jurkat cells pretreated with media $(P<0.05)$. (b) HMDMs were cocultured with apoptotic Jurkat cells in the presence of media (Control), $10 \% \mathrm{CF}$ sol, $10 \%$ CF sol plus DMP777 (100 $\mu \mathrm{M}), 10 \%$ CF sol plus SJ527 (100 $\mu \mathrm{M})$, or 10\% CF sol plus methyl cellulose (MC). The mean phagocytic index as percentage of control \pm SEM is shown for three to five replicates per group. Control mean phagocytic index: $52.8 \pm 10.2$. ${ }^{*}$ Significantly different from control $(P<0.05)$. (c) HMDMs, apoptotic Jurkat cells, or both were pretreated for 30 minutes with media (Control), $10 \% \mathrm{CF}$ sol, $10 \% \mathrm{CF}$ sol plus DMP777 $(20 \mu \mathrm{M})$, or DMP777 $(20 \mu \mathrm{M})$ alone prior to coculture. Following pretreatment, coculture was done in the presence of DMP777 $(20 \mu \mathrm{M})$ to prevent carryover of elastase activity. The mean phagocytic index as percentage of control \pm SEM is shown for three replicates per group. Control mean phagocytic index: $51.4 \pm 6.0 .{ }^{*}$ Significantly different from control $(P<0.05)$. * Significantly different from $10 \%$ CF sol-pretreated HMDMs $(P<0.05) . * *$ Significantly different from $10 \%$ CF sol-pretreated HMDMs and apoptotic Jurkat cells $(P<0.05)$.

decreased the phagocytic index during coculture with apoptotic Jurkat cells $(8.5 \pm 3.0)$ compared with control $(22.5 \pm 5.5)$, but not following preincubation of sol with the natural serine protease inhibitor, $\alpha 1$-antitrypsin, at $100 \mu \mathrm{g} / \mathrm{ml}(15.8 \pm 0.3)$ or $1,000 \mu \mathrm{g} / \mathrm{ml}(16.5 \pm 2.5)$.

Experiments were performed to establish whether the effect of CF sol on apoptotic cell phagocytosis was due to action on the phagocyte, apoptotic cell, or both (Figure 2c). These data showed that CF sol decreased ingestion of apoptotic cells following pretreatment of HMDMs, apoptotic Jurkat cells, or both. DMP777 par- tially prevented CF sol from impairing phagocytosis for all three groups tested, but in the case of apoptotic Jurkat cells statistical significance was not reached. We also tested the possibility that CF HMDMs were intrinsically defective at ingestion of apoptotic cells. However, these experiments did not demonstrate any deficiency in the ability of HMDMs from CF patients to ingest apoptotic cells compared with normal HMDMs (data not shown).

These data suggest that $\mathrm{CF}$ sol diminishes macrophage ingestion of apoptotic cells, but not IgG-opsonized cells, a

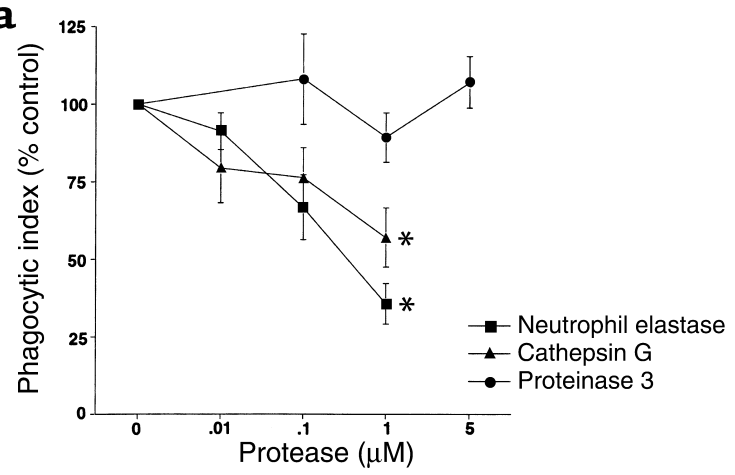

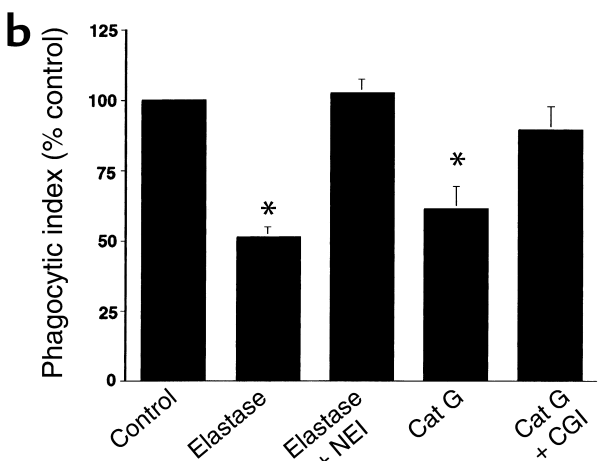

Figure 3

Elastase, or cathepsin G, inhibits ingestion of apoptotic cells. (a) HMDMs were cocultured with apoptotic Jurkat cells in the presence of media or increasing concentrations of elastase, cathepsin G, or proteinase 3. The mean phagocytic index as percentage of control \pm SEM is shown for three to six replicates per group. Control mean phagocytic index: $44.1 \pm 8.5$. *Significantly different from media $(P<0.05)$. (b) Human AMs were cocultured with apoptotic human PMNs following pretreatment with media (Control), $1 \mu \mathrm{M}$ PMN elastase (NE) with or without a specific elastase inhibitor (NEl, $100 \mu \mathrm{M})$, or $1 \mu \mathrm{M}$ cathepsin $\mathrm{G}$ (Cat G) with or without a specific CGI $(10 \mu \mathrm{M})$. Coculture was done in the presence of DMP777 $(20 \mu \mathrm{M})$ to prevent carryover of elastase activity. The mean phagocytic index as percentage of control \pm SEM is shown for three or four replicates per group. Control mean phagocytic index: $8.4 \pm 1.9$. * Significantly different from control $(P<0.05)$. 
a

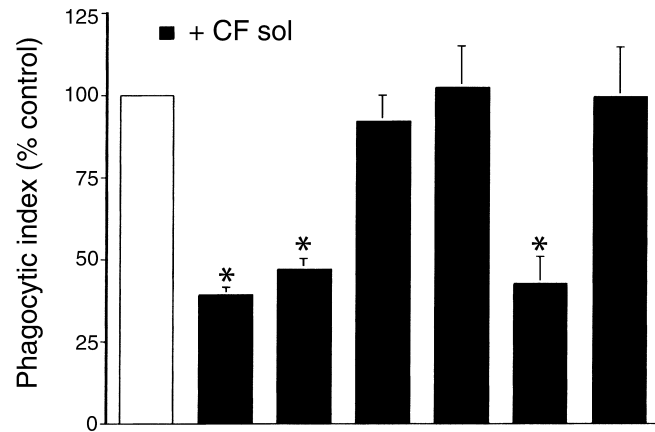

b

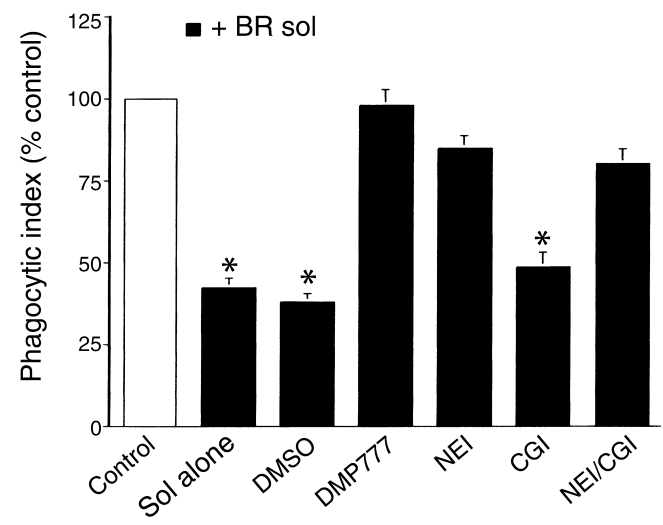

Figure 4

$\mathrm{CF}$ and non-CF bronchiectasis sol-derived elastase disrupts human AM ingestion of apoptotic human PMNs. Human AMs were cocultured with apoptotic human PMNs following pretreatment with media (Control), $10 \%$ CF (a), or $10 \%$ non-CF bronchiectasis (BR) (b) sol with or without DMSO, DMP777 $(20 \mu \mathrm{M})$, NEI $(100 \mu \mathrm{M})$, CGI $(10 \mu \mathrm{M})$, or NEI and CGI. Coculture was done in the presence of DMP777 $(20 \mu \mathrm{M})$ to prevent carryover of elastase activity. The mean phagocytic index as percentage of control \pm SEM is shown for three to four replicates per group. Control mean phagocytic index: (a) $7.9 \pm 1.7$; (b) $7.7 \pm 1.4$. * Groups indicated were significantly different from control $(P<0.05)$.

through an elastase-dependent mechanism. Furthermore, $\mathrm{CF}$ sol appears to reduce phagocytosis of apoptotic cells by acting on both the macrophage and the target apoptotic cell.

PMN elastase and cathepsin G impair macrophage phagocytosis of apoptotic but not IgG-opsonized cells. Three serine proteases with known activity in airway secretions of $\mathrm{CF}$ and non-CF bronchiectasis (elastase, cathepsin G, and proteinase 3 ) were evaluated to determine their effect on HMDM ingestion of apoptotic Jurkat cells (Figure 3a). Elastase $(1 \mu \mathrm{M}, 0.6 \mathrm{U} / \mathrm{ml})$ and cathepsin $\mathrm{G}$ $(1 \mu \mathrm{M}, 70 \mathrm{mU} / \mathrm{ml})$ decreased HMDM ingestion of apoptotic Jurkat cells compared with control. In contrast, proteinase 3 had no effect in the concentrations tested. In addition, $1 \mu \mathrm{M}$ elastase $(13.0 \pm 2.5)$ and $1 \mu \mathrm{M}$ cathepsin G $(9.8 \pm 1.8)$ had no effect on the ability of HMDMs to ingest IgG-opsonized Jurkat cells, compared with control $(9.8 \pm 2.3)$.

In the airway the AM is the professional phagocyte that is most likely to be involved with removal of apoptotic inflammatory cells. Therefore, we sought to test whether serine proteases interfere with human AM ingestion of apoptotic PMNs (Figure 3b). AMs were isolated from normal subjects and were cocultured with apoptotic human PMNs. Elastase $(1 \mu \mathrm{M}, 0.6 \mathrm{U} / \mathrm{ml})$ and cathepsin $\mathrm{G}(1 \mu \mathrm{M}, 70 \mathrm{mU} / \mathrm{ml})$ inhibited human AM uptake of apoptotic human PMNs, and this effect was reversed by the corresponding specific inhibitors. These results confirmed that human AMs ingest apoptotic PMNs and that this uptake is inhibited by either PMN elastase or cathepsin G.

Together, these data establish that elastase and cathepsin G interfere with phagocytosis of apoptotic cells by HMDMs and AMs.

Sol from $C F$ and non-CF bronchiectasis inbibits buman AM ingestion of apoptotic PMNs in an elastase-dependent manner. Experiments were conducted to determine whether solderived serine proteases disrupt the ability of AMs to remove apoptotic PMNs. These experiments demonstrated that sol from CF (Figure 4a) and non-CF bronchiectasis patients (Figure $4 \mathrm{~b}$ ) inhibited human AM clearance of apoptotic human PMNs and that this sol effect was prevented by two specific inhibitors of elastase. In contrast, a specific cathepsin G inhibitor did not, by itself, prevent the effect of sol on apoptotic cell removal. These results strongly suggest that elastase, present in the airways of patients with CF or nonCF bronchiectasis, has the capacity to interfere with removal of apoptotic PMNs by AMs.

$C F$ sol cleaves the PS receptor in an elastase-dependent manner. Flow cytometry was used to test the effect of CF sol on macrophage receptors involved with phagocytosis of apoptotic cells (Figure 5). HMDMs were used in these experiments; however, all receptors tested were also present on human AMs (data not shown). CF sol did not alter staining for CD32 (Fc $\gamma \mathrm{RII})$, CD36, or vitronectin receptor (data not shown). In contrast, $\mathrm{CF}$ sol decreased staining for the PS receptor, but not in the presence of the elastase inhibitor, DMP777. These data suggest that CF sol elastase may interfere with phagocytosis of apoptotic cells through degradation of the PS receptor. To further test this hypothesis, HMDMs were incubated with elastase, cathepsin G, or proteinase 3 , and staining for the PS receptor and CD36 was assessed (Figure 6). Only elastase cleaved the PS receptor at the concentrations tested.

CF sol elastase impairs PS receptor-mediated phagocytosis of apoptotic cells. To test the effect of CF sol on PS receptor-mediated phagocytosis, the PLB985 myelomonocytic cell line was used. PLB985 cells undergo the typical nuclear morphologic and biochemical changes characteristic of apoptosis (7). However, undifferentiated PLB985 cells do not externalize PS from the inner to the outer membrane leaflet during apoptosis (7). Consequently, they do not interact with PS receptor on the surface of the macrophage to drive ingestion. Reconstitution of the plasma membrane outer leaflet with PS, but not PC, increases the ability of macrophages to recognize and ingest apoptotic PLB985 cells through specific interaction with the PS receptor (7). 

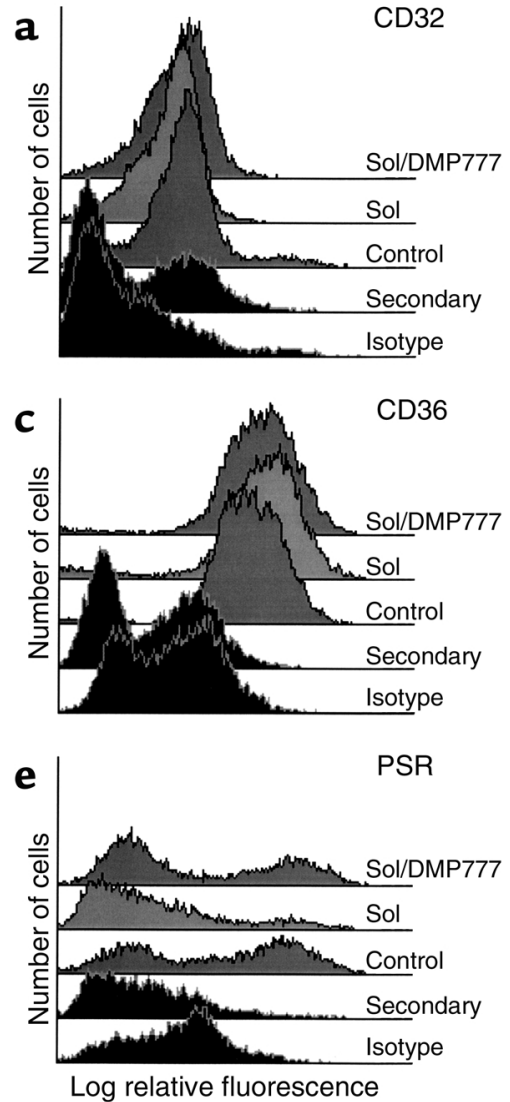

Log relative fluorescence b

d

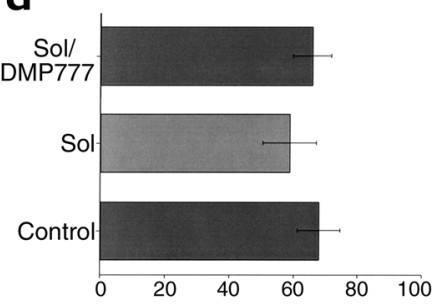

f

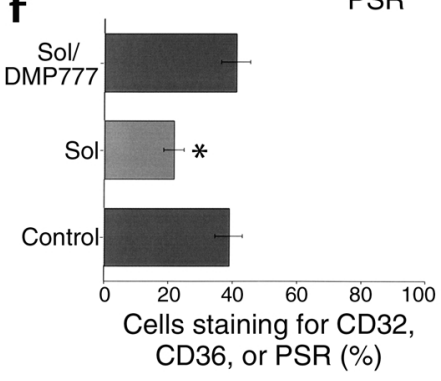

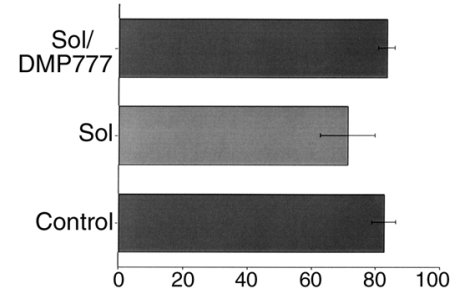

\section{Figure 5}

CF sol cleaves PS receptor (PSR) in an elastase-dependent manner. HMDMs were incubated with media (Control), $10 \%$ CF sol, or $10 \%$ CF sol plus DMP777 $(20 \mu \mathrm{M})$ for 2 hours. Flow cytometry was done to assess surface staining for CD32 (a and b), CD36 ( $\mathbf{c}$ and $\mathbf{d}$ ), and PSR (e and $\mathbf{f}$ ). Histograms for each receptor are shown in the left panels, and the percent of HMDMs that stain for each receptor \pm SEM are shown in the right panels (four to six replicates per group). *Significantly different from control $(P<0.05)$.

ance of apoptotic cells might be abnormal. Our data indicate that apoptotic cells are present in the sputa from all three groups studied, but are much more abundant in patients with CF and non-CF bronchiectasis, compared with those with chronic bronchitis. A variety of potential mechanisms may contribute to accumulation of apoptotic inflammatory cells in these circumstances. However, data presented here suggest that airway elastase inhibits apoptotic cell clearance through direct action on macrophage PS receptors. The finding of a large reservoir of uncleared, dying inflammatory cells may have a significant impact on ongoing airway inflammation.

Increased apoptotic cells in the airways of patients with CF and non-CF bronchiecta-
The effect of CF sol on phagocytosis of apoptotic cells through the PS receptor was tested using viable and apoptotic PLB985 cells (Figure 7). In some PLB985 cells, membrane PS, or control PC, was reconstituted prior to coculture. Reconstitution of membrane PS, but not PC, increased HMDM ingestion of apoptotic PLB985cells, compared with viable PLB985 cells. In contrast, PS reconstitution of apoptotic cells did not augment their engulfment by CF sol-pretreated HMDMs, except in the presence of DMP777. Therefore, these results imply that degradation of PS receptor by $\mathrm{CF}$ sol elastase directly impairs apoptotic cell clearance by HMDMs.

\section{Discussion}

Three human diseases characterized by ated to determine whether airway clear-

\section{Figure 6}

Elastase, but not cathepsin G or proteinase 3, cleaves cell surface PSR. HMDMs were incubated with media proteinase $3(1 \mu \mathrm{M})$ for 2 hours. Flow cytometry was done to assess surface staining for PS receptor ( $\mathbf{a}$ and b) and CD36 (c and d). Histograms for each receptor are shown in the left panels, and the percent of HMDMs that stain for each receptor \pm SEM are shown in the right panels (three replicates per group). ${ }^{*}$ Significantly different from control $(P<0.05)$. chronic airway inflammation were evalu(Control), elastase $(1 \mu \mathrm{M})$, cathepsin $\mathrm{G}(1 \mu \mathrm{M})$, or a
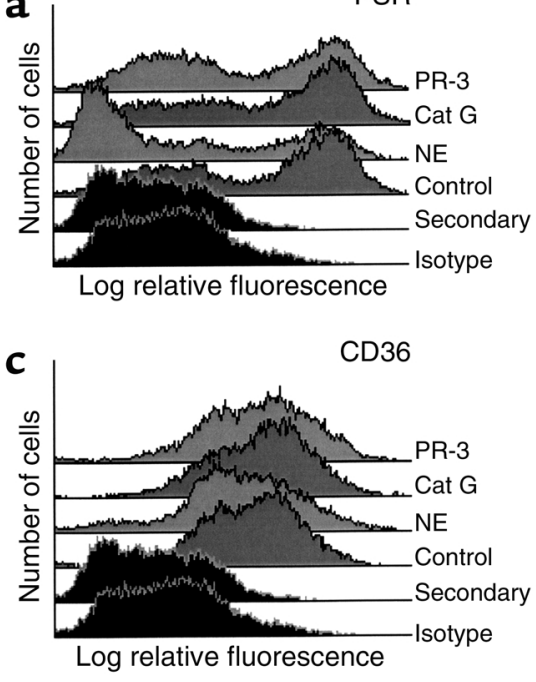

b
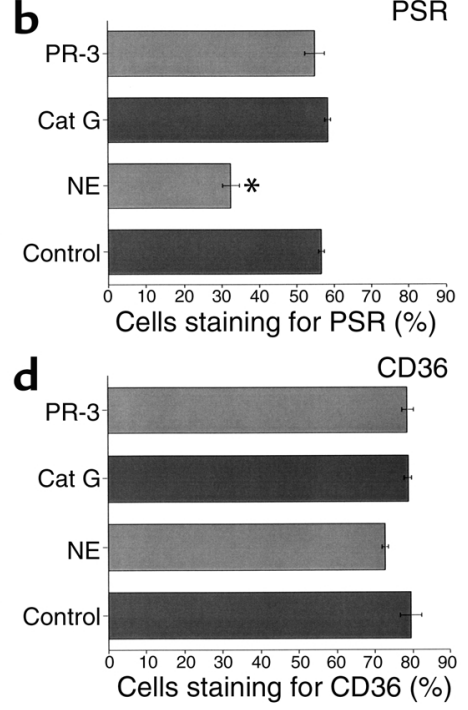
sis suggest that the normal kinetics of apoptotic cell clearance is impaired, since in other PMN-dominated inflammatory pulmonary diseases efficient clearance results in very low numbers of apoptotic cells $(18,20)$. The airway PMN burden in the acute respiratory distress syndrome (18) and community-acquired pneumonia (19) is similar to that found in clinically stable CF patients $\left(1.9 \times 10^{5} / \mathrm{ml}\right)$ and in CF patients with clinical exacerbations $\left(1.3 \times 10^{6} / \mathrm{ml}\right)(29)$. However, in 


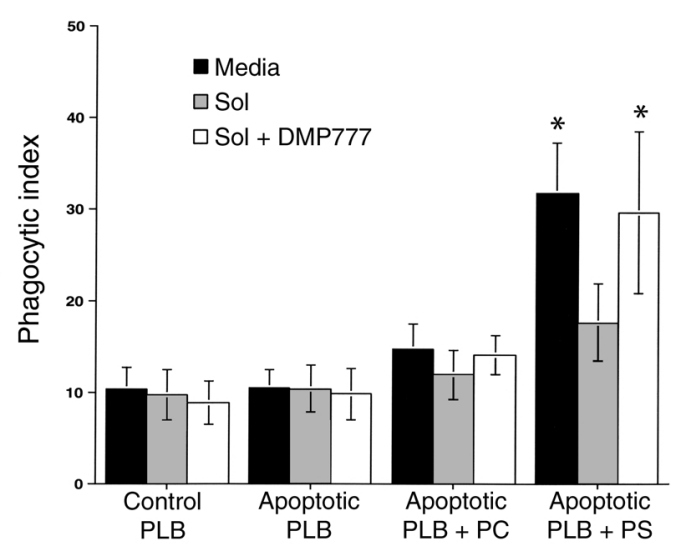

Figure 7

CF sol interferes with PS-mediated apoptotic cell ingestion in an elastase-dependent manner. HMDMs were pretreated with media, 10\% CF sol, or $10 \%$ CF sol plus DMP777 $(20 \mu \mathrm{M})$ for 30 minutes prior to coculture with viable or apoptotic PLB985 cells. PS or PC was inserted into the outer membrane of some apoptotic PLB985 cells by phospholipid transfer. HMDMs were then cocultured for 1 hour with viable PLB985 cells (Control), apoptotic PLB985 cells, apoptotic PLB985 cells plus PC, or apoptotic PLB985 cells plus PS. Coculture was done in the presence of DMP777 $(20 \mu \mathrm{M})$ to prevent carryover of elastase activity. The mean phagocytic index \pm SEM is shown for five replicates per group. *Significantly different from control $(P<0.05)$.

contrast to the acute respiratory distress syndrome and community-acquired pneumonia, where less than $2 \%$ of lavage cells are reported to be apoptotic $(18,20)$, we found that more than $25 \%$ of inflammatory cells in CF sputum were apoptotic, $19 \%$ were apoptotic in bronchiectasis, and $3 \%$ were apoptotic in chronic bronchitis. This observation suggests that factors unique to $\mathrm{CF}$, bronchiectasis, and possibly chronic bronchitis, may impair the normal apoptotic cell clearance mechanisms.

Since CF and non-CF bronchiectasis airway lining fluid significantly blocked uptake of apoptotic cells into macrophages, inhibitory materials in these airways were considered prime candidates for the effect. However, alternate explanations for apoptotic cell accumulation abound and should be considered. (a) We do not believe that the observed increase in apoptotic inflammatory cells is primarily a function of accelerated apoptosis. We found that peripheral blood PMNs from CF patients were not defective at spontaneous or ultraviolet-induced apoptosis in vitro, measured by morphology and PS expression (data not shown). The local airway environment (e.g., hyperosmolarity) could increase the rate of PMN apoptosis (30). However, available evidence strongly suggests that massive apoptosis can be cleared expediently using normal clearance mechanisms (1). Therefore, accelerated apoptosis by any means seems unlikely to account, solely, for the observed accumulation of apoptotic cells. (b) Entrapment of apoptotic cells within airway mucus and/or altered clearance by an impaired mucociliary escalator may well contribute to decreased clearance. However, biopsies of CF airways have demonstrated the presence of apoptotic cells in the airway walls, suggesting that these factors are not necessary for apoptotic cells to accumulate $(31,32)$. (c) Since most cell types in the body, including epithelial cells $(8,33)$, can ingest apoptotic cells, there may be defects in clearance at the level of the airway epithelium related to altered CF transmembrane regulator function. This might be especially relevant to early inflammation in CF. However, by the time inflammatory processes have become persistent, much of the clearance is more likely due to alveolar macrophages. Our studies of monocyte-derived macrophages from individuals with CF showed no intrinsic abnormalities in apoptotic cell engulfment (data not shown). (d) Finally, observed decreases in the lung collectins, such as surfactant protein A and surfactant protein D (34), may also contribute to poor apoptotic cell clearance, since collectins have been implicated in apoptotic cell removal (14).

The experiments described herein suggest that the airway lining fluid contains proteases that are capable of inhibiting apoptotic cell clearance, in particular elastase and cathepsin G. A variety of proteases are present in CF airway lining fluid, including elastase $(23,28,35)$, cathepsin $\mathrm{G}(28,36)$, proteinase 3 (37), bacterial proteinases, such as Pseudomonas elastase (38), and metalloproteinases (39). While any one of these proteases potentially may interfere with apoptotic cell phagocytosis, we tested the PMN serine proteases since they are the most abundant. These studies demonstrated that $\mathrm{CF}$ and non-CF bronchiectasis sol contained elastase and cathepsin $\mathrm{G}$ in high concentrations, similar to those measured by others $(28,35)$. However, $\mathrm{CF}$ and non-CF bronchiectasis sol only impaired the ability of macrophages to ingest apoptotic cells in an elastasedependent manner. When tested individually, purified elastase and cathepsin $\mathrm{G}$ both inhibited macrophage uptake of apoptotic cells, whereas proteinase 3 did not. This observed difference in the effectiveness of solderived cathepsin $G$ versus purified cathepsin $G$ can likely be explained by the lower activity of cathepsin $\mathrm{G}$ found in $10 \% \mathrm{CF}$ sol $(2.8 \mathrm{mU} / \mathrm{ml})$ and $10 \%$ non-CF bronchiectasis sol $(7.7 \mathrm{mU} / \mathrm{ml})$ compared with the activity of purified cathepsin $\mathrm{G}(70 \mathrm{mU} / \mathrm{ml})$ that was necessary to inhibit apoptotic cell uptake. In contrast, the concentration of purified elastase $(0.6 \mathrm{U} / \mathrm{ml})$ necessary to inhibit apoptotic cell uptake was similar to that found in $10 \% \mathrm{CF}$ sol $(0.3 \mathrm{U} / \mathrm{ml})$ and $10 \%$ non-CF bronchiectasis sol $(1.0 \mathrm{U} / \mathrm{ml})$. While we feel that the primary effect of sol is on the macrophage, our data also establishes that CF sol disrupts clearance by acting directly on the apoptotic cell. Apoptotic cell targets for this CF sol effect remain obscure; however, possibilities include protease cleavage of glycoprotein ligands or direct blockade of externalized PS. This effect of sol proteases is consistent with our current knowledge of the protease/antiprotease imbalance that exists in CF and non-CF bronchiectasis airways, where proteases are present in excess of their major inhibitors $(23,24)$. 
Since protease/antiprotease imbalance is also a feature of chronic bronchitis (25), we would anticipate that chronic bronchitis sol would also impair clearance of apoptotic cells, but perhaps to a lesser extent than $\mathrm{CF}$ or non-CF bronchiectasis sol.

Unopposed airway proteases have been shown to inflict significant damage to host defense by injuring bronchial epithelial cells, destroying extracellular matrix components, cleaving immunoglobulin, complement, and fibronectin (23). Serine proteases may also cleave cell surface molecules and impair a variety of cell functions including phagocytosis. For example, elastase inhibits PMN phagocytosis of $P$. aeruginosa by cleaving both CR1 and C3bi, creating an "opsonin receptor mismatch" (40). Elastase also cleaves cell surface antigens (CD4 and CD8), causing impaired $\mathrm{T}$ lymphocyte-mediated cytotoxicity (41), and cathepsin G degrades CD14 on monocytes (42). Our data suggest that elastase in CF and non-CF bronchiectasis sol interferes with phagocytosis through cleavage of macrophage surface PS receptor. The PS receptor is a recently cloned, small, transmembrane phagocyte receptor, present on HMDMs (8, 43) and AMs (data not shown), that drives ingestion of apoptotic cells through stereospecific recognition of PS on the outer surface of apoptotic cells. It possesses potential elastase cleavage sites near the transmembrane domain that would result in loss of the putative PSbinding region of the molecule (8). The mechanism by which cathepsin $\mathrm{G}$ interferes with apoptotic cell ingestion remains unknown; however, cleavage of CD14, a receptor known to be involved with apoptotic cell removal, is one possibility. Alternatively, the PS receptor may be cleaved by cathepsin $\mathrm{G}$, but cleavage may not be detected by the techniques used.

Since removal of dying inflammatory cells is an integral step in the resolution of inflammation, failure of this process may have proinflammatory consequences in the airways of patients with $\mathrm{CF}$ and non-CF bronchiectasis. Recognition of apoptotic cells by phagocytes through the PS receptor is actively antiinflammatory, inducing TGF- $\beta$, prostaglandin $\mathrm{E}_{2}(4)$, and under some circumstances, IL-10 (5); these may act in an autocrine/paracrine manner to suppress production of a host of proinflammatory cytokines (TNF- $\alpha$ ), chemokines (IL-8), growth factors, and lipid mediators $(4,5)$, similar to inflammatory mediators that are increased in CF airways $(21,22)$. Cleavage of the PS receptor would be expected to block this effect. In contrast, macrophage removal of lysed PMNs is much less effective at inducing TGF- $\beta$ and inhibiting inflammatory mediators, in part because of its content of elastase (44). Evidence was presented here that PMNs in CF airways were also undergoing postapoptotic necrosis. This would be expected to eventually cause release of intracellular contents with potent inflammatory potential, including the elastase proposed to participate in blockade of the apoptotic cell removal.

This concept raises a potential paradox. In the normal acute inflammatory response, early influx of
PMNs might well be expected to result in some release of elastase $(19,45)$ (even though extracellular elastase secretion is, in fact, not a major event in PMN activation). Cleavage of the PS receptor would limit production of anti-inflammatory cytokines. Why then, do all acute inflammatory reactions not progress? It would seem likely that the key limiting factor is the availability (influx) of functional antiproteases, which in a "normal" inflammatory reaction would bring the protease balance back to normal, allow PS receptor-mediated generation of anti-inflammatory mediators, appropriate clearance of the apoptotic inflammatory cells, and resolution of the response.

These results suggest that impaired clearance of apoptotic cells in diseases characterized by protease/antiprotease imbalance contributes to the enhanced inflammatory response and may precipitate ongoing inflammation and progressive airway damage. Therefore, therapies directed toward relieving this imbalance may diminish progressive pulmonary decline by preventing accumulation of apoptotic cells, subsequent postapoptotic necrosis, and defective resolution of inflammation.

\section{Acknowledgments}

We thank G. Smith and J. Henson for technical assistance with transmission electron microscopy, D. Riches for advice and discussion, J. Nick for critical review of the manuscript, and DuPont Pharmaceuticals Co. for kindly providing DMP777. Grants from the Cystic Fibrosis Foundation (to R.W. Vandivier) and the NIH (GM-48211 and HL-60980 to P.M. Henson) supported this work.

1. Wyllie, A.H., Kerr, J.F., and Currie, A.R. 1980. Cell death: the significance of apoptosis. Int. Rev. Cytol. 68:251-306.

2. Savill, J.S., et al. 1989.Macrophage phagocytosis of aging neutrophils in inflammation. Programmed cell death in the neutrophil leads to its recognition by macrophages. J. Clin. Invest. 83:865-875.

3. Lethem, M.I., James, S.L., Marriott, C., and Burke, J.F. 1990. The origin of DNA associated with mucus glycoproteins in cystic fibrosis sputum. Eur. Respir. J. 3:19-23.

4. Fadok, V.A., et al. 1998. Macrophages that have ingested apoptotic cells in vitro inhibit proinflammatory cytokine production through autocrine/paracrine mechanisms involving TGF-beta, $\mathrm{PGE}_{2}$, and PAF. J. Clin. Invest. 101:890-898.

5. McDonald, P.P., Fadok, V.A., Bratton, D., and Henson, P.M. 1999. Transcriptional and translational regulation of inflammatory mediator production by endogenous TGF-beta in macrophages that have ingested apoptotic cells. J. Immunol. 163:6164-6172.

6. Fadok, V.A., et al. 1992. Exposure of phosphatidylserine on the surface of apoptotic lymphocytes triggers specific recognition and removal by macrophages. J. Immunol. 148:2207-2216.

7. Fadok, V.A., de Cathelineau, A., Daleke, D.L., Henson, P.M., and Bratton, D.L. 2001. Loss of phospholipid asymmetry and surface exposure of phosphatidylserine is required for phagocytosis of apoptotic cells by macrophages and fibroblasts. J. Biol. Chem. 276:1071-1077.

8. Fadok, V.A., et al. 2000. A receptor for phosphatidylserine-specific clearance of apoptotic cells. Nature. 405:85-90.

9. Savill, J., Hogg, N., Ren, Y., and Haslett, C. 1992. Thrombospondin cooperates with CD36 and the vitronectin receptor in macrophage recognition of neutrophils undergoing apoptosis. J. Clin. Invest. 90:1513-1522.

10. Platt, N., Suzuki, H., Kodama, T., and Gordon, S. 2000. Apoptotic thymocyte clearance in scavenger receptor class A-deficient mice is apparently normal. J. Immunol. 164:4861-4877.

11. Devitt, A., et al. 1998. Human CD14 mediates recognition and phagocytosis of apoptotic cells. Nature. 392:505-509.

12. Savill, J., Dransfield, I., Hogg, N., and Haslett, C. 1990.Vitronectin recep- 
tor-mediated phagocytosis of cells undergoing apoptosis. Nature. 343:170-173.

13. Albert, M.L., Kim, J.I., and Birge, R.B. 2000. Alphavbeta5 integrin recruits the CrkII-Dock180-rac1 complex for phagocytosis of apoptotic cells. Nat. Cell Biol. 2:899-905.

14. Schagat, T.L., Wofford, J.A., and Wright, J.R. 2001. Surfactant protein A enhances alveolar macrophage phagocytosis of apoptotic neutrophils. J. Immunol. 166:2727-2733.

15. Ogden, C.A., et al. 2001. C1q and mannose binding lectin engagement of cell surface calreticulin and CD91 initiates macropinocytosis and uptake of apoptotic cells. J. Exp. Med. 194:781-796.

16. Taylor, P.R., et al. 2000. A hierarchical role for classical pathway complement proteins in the clearance of apoptotic cells in vivo. J. Exp. Med. 192:359-366.

17. Huynh, M.-L.N., Fadok, V.A., and Henson, P.M. 2002. Phosphatidylserine-dependent ingestion of apoptotic cells promotes TGF- $\beta 1$ secretion and the resolution of inflammation. J. Clin. Invest. 109:41-50. DOI:10.1172/JCI200211638.

18. Matute-Bello, G., et al. 1997. Neutrophil apoptosis in the acute respiratory distress syndrome. Am. J. Respir. Crit. Care Med. 156:1969-1977.

19. Boutten, A., et al. 1996. Compartmentalized IL-8 and elastase release within the human lung in unilateral pneumonia. Am. J. Respir. Crit. Care Med. 153:336-342.

20. Droemann, D., et al. 2000. Decreased apoptosis and increased activation of alveolar neutrophils in bacterial pneumonia. Chest. 117:1679-1684

21. Khan, T.Z., et al. 1995. Early pulmonary inflammation in infants with cystic fibrosis. Am. J. Respir. Crit. Care Med. 151:1075-1082.

22. Bonfield, T.L., et al. 1995. Inflammatory cytokines in cystic fibrosis lungs [erratum 1996, 154:1217]. Am. J. Respir. Crit. Care Med. 152:2111-2118.

23. Birrer, P., et al. 1994. Protease-antiprotease imbalance in the lungs of children with cystic fibrosis. Am. J. Respir. Crit. Care Med. 150:207-213.

24. Tsang, K.W., et al. 2000. Sputum elastase in steady-state bronchiectasis. Chest. 117:420-426.

25. Stockley, R.A. 1999. Neutrophils and protease/antiprotease imbalance. Am. J. Respir. Crit. Care Med. 160(Suppl.):S49-S52.

26. Vignola, A.M., et al. 1998. Increased levels of elastase and alpha1-antitrypsin in sputum of asthmatic patients. Am. J. Respir. Crit. Care Med. 157:505-511.

27. Turino, G.M., et al. 1969. Serum elastase inhibitor deficiency and alpha 1-antitrypsin deficiency in patients with obstructive emphysema. Science. 165:709-711.

28. Rees, D.D., and Brain, J.D. 1995. Effects of cystic fibrosis airway secretions on rat lung: role of neutrophil elastase. Am. J. Physiol. 269:L195-L202.

29. Meyer, K.C., et al. 1991. Human neutrophil elastase and elastase/alpha 1 -antiprotease complex in cystic fibrosis. Comparison with interstitial lung disease and evaluation of the effect of intravenously administered antibiotic therapy. Am. Rev. Respir. Dis. 144:580-585.

30. Tager, A.M., Wu, J., and Vermeulen, M.W. 1998. The effect of chloride concentration on human neutrophil functions: potential relevance to cystic fibrosis. Am. J. Respir. Cell Mol. Biol. 19:643-652.

31. Maiuri, L., et al. 1997. DNA fragmentation is a feature of cystic fibrosis epithelial cells: a disease with inappropriate apoptosis? FEBS Lett. 408:225-231.

32. Durieu, I., et al. 1999. Fas and Fas ligand expression in cystic fibrosis airway epithelium. Thorax. 54:1093-1098.

33. Walsh, G.M., Sexton, D.W., Blaylock, M.G., and Convery, C.M. 1999. Resting and cytokine-stimulated human small airway epithelial cells recognize and engulf apoptotic eosinophils. Blood. 94:2827-2835.

34. Postle, A.D., et al. 1999. Deficient hydrophilic lung surfactant proteins A and D with normal surfactant phospholipid molecular species in cystic fibrosis. Am. J. Respir. Cell Mol. Biol. 20:90-98.

35. Liu, H., Lazarus, S.C., Caughey, G.H., and Fahy, J.V. 1999. Neutrophil elastase and elastase-rich cystic fibrosis sputum degranulate human eosinophils in vitro. Am. J. Physiol. 276:L28-L34.

36. Goldstein, W., and Doring, G. 1986. Lysosomal enzymes from polymorphonuclear leukocytes and proteinase inhibitors in patients with cystic fibrosis. Am. Rev. Respir. Dis. 134:49-56.

37. Witko-Sarsat, V., et al. 1999. Proteinase 3, a potent secretagogue in airways, is present in cystic fibrosis sputum. Am. J. Respir. Cell Mol. Biol. 20:729-736.

38. Suter, S. 1994. The role of bacterial proteases in the pathogenesis of cystic fibrosis. Am. J. Respir. Crit. Care Med. 150(Suppl.):S118-S122.

39. Delacourt, C., et al. 1995. Imbalance between $95 \mathrm{kDa}$ type IV collagenase and tissue inhibitor of metalloproteinases in sputum of patients with cystic fibrosis. Am. J. Respir. Cell Med. 152:765-774.

40. Tosi, M.F., Zakem, H., and Berger, M. 1990. Neutrophil elastase cleaves C3bi on opsonized pseudomonas as well as CR1 on neutrophils to create a functionally important opsonin receptor mismatch. J. Clin. Invest. 86:300-308.

41. Doring, G., et al. 1995. Cleavage of lymphocyte surface antigens CD2, $\mathrm{CD} 4$, and CD8 by polymorphonuclear leukocyte elastase and cathep$\sin \mathrm{G}$ in patients with cystic fibrosis. J. Immunol. 154:4842-4850.

42. Le-Barillec, K., Pidard, D., Balloy, V., and Chignard, M. 2000. Human neutrophil cathepsin G down-regulates LPS-mediated monocyte activation through CD14 proteolysis. J. Lenkoc. Biol. 68:209-215.

43. Hu, B., Sonstein, J., Christensen, P.J., Punturieri, A., and Curtis, J.L. 2000 . Deficient in vitro and in vivo phagocytosis of apoptotic T cells by resident murine alveolar macrophages. J. Immunol. 165:2124-2133.

44. Fadok, V.A., Bratton, D.L., Guthrie, L., and Henson, P.M. 2001. Differential effects of apoptotic versus lysed cells on macrophage production of cytokines: role of proteases. J. Immunol. 166:6847-6854.

45. Cochrane, C.G., Spragg, R.G., Revak, S.D., Cohen, A.B., and McGuire, W.W. 1983. The presence of neutrophil elastase and evidence of oxidation activity in bronchoalveolar lavage fluid of patients with adult respiratory distress syndrome. Am. Rev. Respir. Dis. 127(Suppl.):S25-S27. 